UCRL-JC-121954

PREPRINT

\title{
Isoelectronic Behavior of Resonant and Intercombination Lines in MgI-Like Ions
}

\author{
K. B. Fournier \\ W. H. Goldstein \\ M. Finkenthal \\ R. E. Bell \\ J. L. Terry
}

This paper was prepared for submittal to

11th International Conference on Vacuum Ultraviolet Radiation Physics

Rikkyo University, Tokyo

August 27 to September 1, 1995

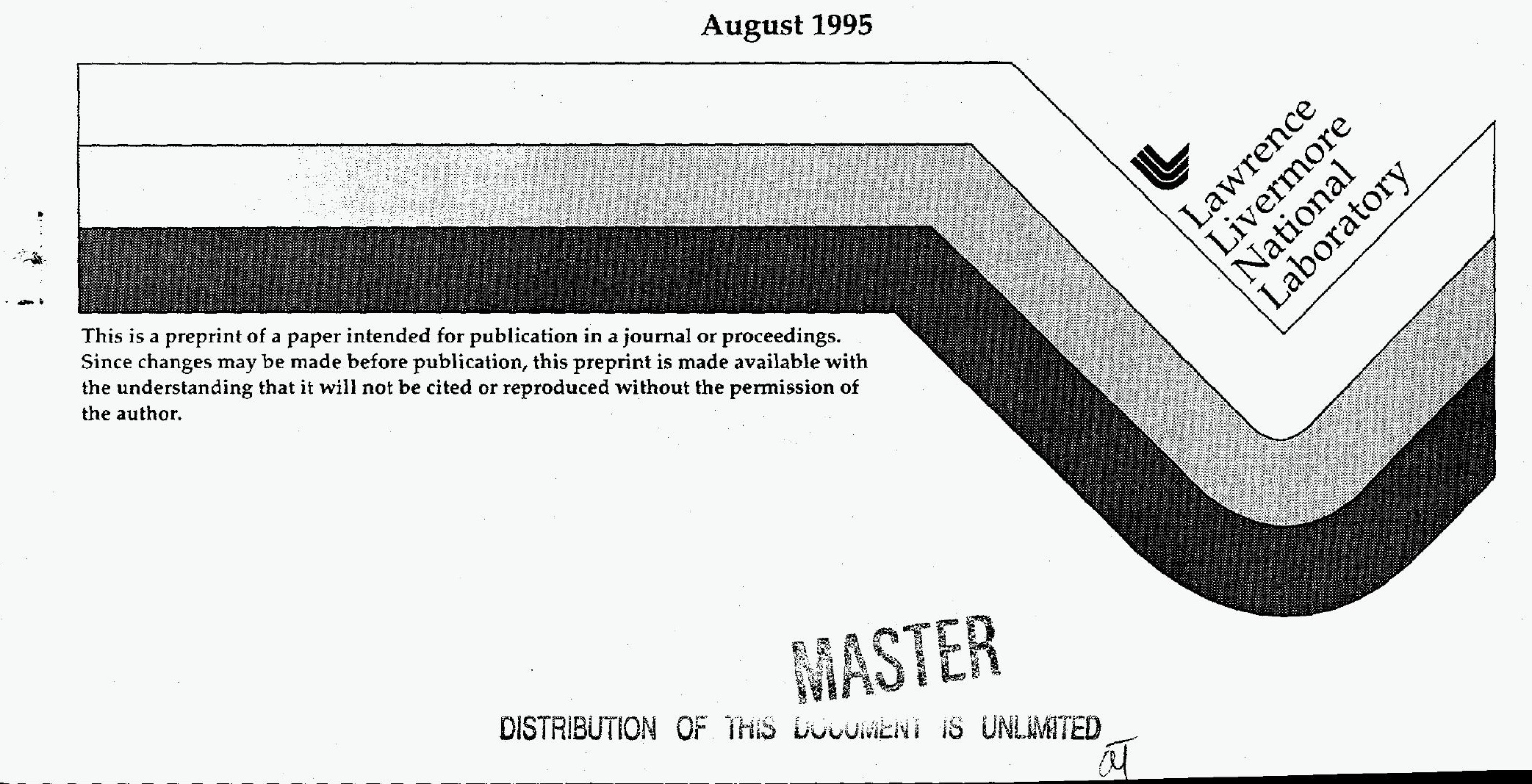




\section{DISCLAIMER}

This document was prepared as an account of work sponsored by an agency of the United States Government. Neither the United States Government nor the University of California nor any of their employees, makes any warranty, express or implied, or assumes any legal liability or responsibility for the accuracy, completeness, or usefulness of any information, apparatus, product, or process disciosed, or represents that its use would not infringe privately owned rights. Reference herein to any specific commercial product, process, or service by trade name, trademark, manufacturer, or otherwise, does not necessarily constitute or imply its endorsement, recommendation, or favoring by the United States Government or the University of California. The views and opinions of authors expressed herein do not necessarily state or reflect those of the United States Government or the University of California, and shall not be used for advertising or product endorsement purposes. 


\section{DISCLAIMER}

Portions of this document may be illegible in electronic image products. Images are produced from the best available original document. 


\title{
Isoelectronic Behavior of Resonant and Intercombination Lines in MgI-like Ions
}

\author{
K. B. Fournier ${ }^{a *}$, W. H. Goldstein ${ }^{a}$, M. Finkenthal ${ }^{b \dagger}$, R. E. Bell ${ }^{c}$ and J. L. Terry ${ }^{d}$

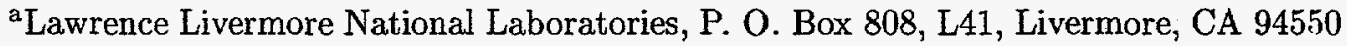 \\ b Johns Hopkins University, Department of Physics and Astronomy, Baltimore, MD 21218 \\ 'Princeton Plasma Physics Laboratory, Princeton University, Princeton, NJ 08543

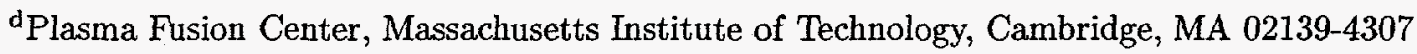

The observed intensity ratio of the $3 s^{2}{ }^{1} S_{0}-3 s 3 p{ }^{1} P_{1}$ to the $3 s^{2}{ }^{1} S_{0}-3 s 3 p{ }^{3} P_{1}$ transitions in MgI-like ions has always presented those who nodel plasma spectra with a challenge; the observed intensity of the intercombination line is always several times greater than what simple models predict. We offer a model that takes into account the contribution to the MgI-like emission features from autoionizing levels of the adjacent AlI-like charge state. Models in the present work, which include the effects of configuration interaction on ionic wavefunctions, and the contribution of both direct, impact ionization and autoionization channels from the AlI-like ion to the MgI-like ion, give good agreement with the observed resonant/intercombination (R/I) emission ratio only when a departure from ionization equilibrium is assumed. We also identify, for the first time, intercombination lines of the form $3 s 3 p{ }^{1} P_{1}-3 p^{2}{ }^{3} P_{2}$ in several elements relevant to both astrophysical and magnetically-confined fusion plasmas.

\section{Introduction}

Radiative transitions with very different characteristic rates can serve as important diagnostics of local conditions in a plasma. The $3 s^{2}{ }^{1} \mathrm{~S}_{0}-3 \mathrm{~s} 3 \mathrm{p}$ ${ }^{1} \mathrm{P}_{1}$ resonant line and $3 \mathrm{~s}^{2}{ }^{1} \mathrm{~S}_{0}-3 \mathrm{~s} 3 \mathrm{p}{ }^{3} \mathrm{P}_{1}$ intercombination line of $\mathrm{MgI}-$ like ions have transition rates that differ by orders-of-magnitude; the difference in transition rates means the slower transition will be strongly quenched by collisions relative to the faster transition as the local electron density changes. The use of high- $Z$ elements in several magnetically-confined fusion experiments (e.g. Alcator C-Mod tokamak, Frascati Tokamak Upgrade), and the presence of high- $Z$ dopants in laser-produced plasmas are but two motivations for the study of the kinetics of highly ionized, high-Z materials. For astrophysical plasmas, like the solar corona, VUV- and XUV-observations of ionic line radiation are often the only source of information about the local plasma conditions.

The FeXV $3 s^{2}{ }^{1} S_{0}-3 s 3 p{ }^{3} P_{1}$ intercombination line has been studied in spectra taken of the solar corona during flare events [1] and in the quiet solar corona [2]. Attempts to explain

\footnotetext{
*The Johns Hopkins University

†Permanent Address: Racah Institute of Physics, The Hebrew University, Jerusalem, Israel
}

the observed ratio of the emissivity of the FeXV $3 \mathrm{~s}^{2}{ }^{1} \mathrm{~S}_{0}-3 \mathrm{~s} 3 \mathrm{p}{ }^{3} \mathrm{P}_{1}$ intercombination line to the emissivity of the $3 \mathrm{~s}^{2}{ }^{1} \mathrm{~S}_{0}-3 \mathrm{~s} 3 \mathrm{p}{ }^{1} \mathrm{P}_{1}$ resonant line $(\mathrm{R} / \mathrm{I})$ were made by Bhatia and Kastner [3]. Bhatia and Kastner offered the inclusion of the 3s3d and $3 \mathrm{p}^{2}$ excited configurations in the collisionalradiative model to account for both radiative cascades from excited levels and the effect on collision strengths from wavefunction mixing. Christensen, Norcross and Pradhan [4] (CNP) found the $R$-matrix collision strengths from the ground state of MgI-like iron to the levels of the 3s3p excited configuration to be a factor of two greater than the DWA-collision strengths used by earlier researchers. Dufton, Kingston and Widing [5] (DKW) discuss the kinetics of the FeXV spectrum. One important result in DKW is that the $R$-matrix collision strengths of CNP do not effect the FeXV R/I ratio as much as might be expected; the narrowness of the resonance region compared to the temperature at which FeXV is expected to exist means the collision-rates from the ground state to the levels of the $3 \mathrm{~s} 3 \mathrm{p}$ configuration are enhanced by at most $10 \%$.

A model which uses innershell ionization from the 3s-subshell of the AlI-like ion to explain the observed $\mathrm{R} / \mathrm{I}$ line ratio in $\mathrm{MgI}-$ like selenium was offered by Finkenthal et al. [6]. There, Finken- 
thal et al. needed twice as much of the AlI-like ion as the MgI-like ion present to reproduce the experiment. Feldman et al. [7] also used an innershell ionization model to explain the observed $\mathrm{R} / \mathrm{I}$ line ratio in solar iron spectra. The conclusion reached by Feldman et al. is that the mechanism of innershell ionization could explain the observed line ratio only if the FeXV and FeXIV were at temperatures far above their expected coronal ionization equilibrium temperature, or if there were a non-thermal population of free electrons doing (some of) the ionization. The first observation of the FeXV intercombination line in a laboratory plasma was made in the TFR tokamak by Finkenthal et al. [8]. The wavelengths and brightness of the resonant and intercombination lines for other astrophysically relevant elements ( $\mathrm{Ti}, \mathrm{V}$ and $\mathrm{Cr}$ ) were also observed in TFR [8]. The resonant and intercombination lines of $\mathrm{MgI}$-like ions of heavier elements (Ge, Se and Mo) have been observed in other tokamak plasmas [9].

\section{Models}

Models for the emission from the MgI-like ion of several elements ( $\mathrm{Ti}, \mathrm{V}, \mathrm{Cr}, \mathrm{Fe}, \mathrm{Ge}, \mathrm{Se}$ and $\mathrm{Mo}$ ) in quasi-steady state (QSS) collisional-radiative (CR) equilibrium have been made. The ground state populations of the AlI-, MgI- and NaI-like charge states of each element are found from the best available models $[10,11]$ and entered into the collisional-radiative rate matrix

$\frac{d n_{j}}{d t}=\sum_{i} n_{i} R_{i \rightarrow j}-n_{j} \sum_{i} R_{j \rightarrow i}$

where $n_{i}$ is the population in level ' $i$ ' of a given ion, and $R_{i \rightarrow j}$ is the rate at which population leaves level ' $i$ ' and goes to some level ' $j$ ', possibly of a neighboring isosequence. Under the QSS approximation [12], $d n_{i} / d t$ is assumed to be zero for all levels but the ground level of each ion; the level populations in the excited states of each ion are then found. The ionization balance models of Arnaud and Raymond [10] for iron and Fournier et al. [11] for molybdenum have been scaled to provide the fractional abundance of each charge state for the other elements at the reported experimental conditions $[8,9]$.

For the MgI-like ions, all levels formed from the $3 L 3 l$ ' and $3 \mathrm{~s} 4 l$ ' configurations, as well as all levels from the $\overline{2 l} 3 s 3 l^{\prime} 3 l^{\prime \prime}$ and $\overline{2 l} 3 s^{2} 4 l^{\prime}$ configurations, where $\overline{2 l}$ indicates a $n=2$ hole, have been in- cluded in the present models. Similar treatment is given to the AlI- and NaI-like ions. The details of this type of model have been presented elsewhere [13]. $A b$ initio atomic structure for the QSS-CR models comes from the HULLAC package $[14,15]$. For all states formed by the promotion of a $n=2$ electron, the rates of autoionization (AI) were computed in the distorted wave approximation by RELAC [16]. The rates of electronimpact ionization, from both the valence shell and the bound, inner subshells were found from employing RELAC's relativistic subshell energies in the Lotz formula [17]. The AI states of the AlI-like ion contribute population to the intercombination line via the $3 \mathrm{p}^{2}{ }^{3} \mathrm{P}_{0}$ level of the MgI-like ion, but much less than the innershell, impact ionization channels. Some of the energy level structure and the key channels for population flux in the MgIlike ions in the present work are shown in Fig 1. (NaI-like levels are not shown).

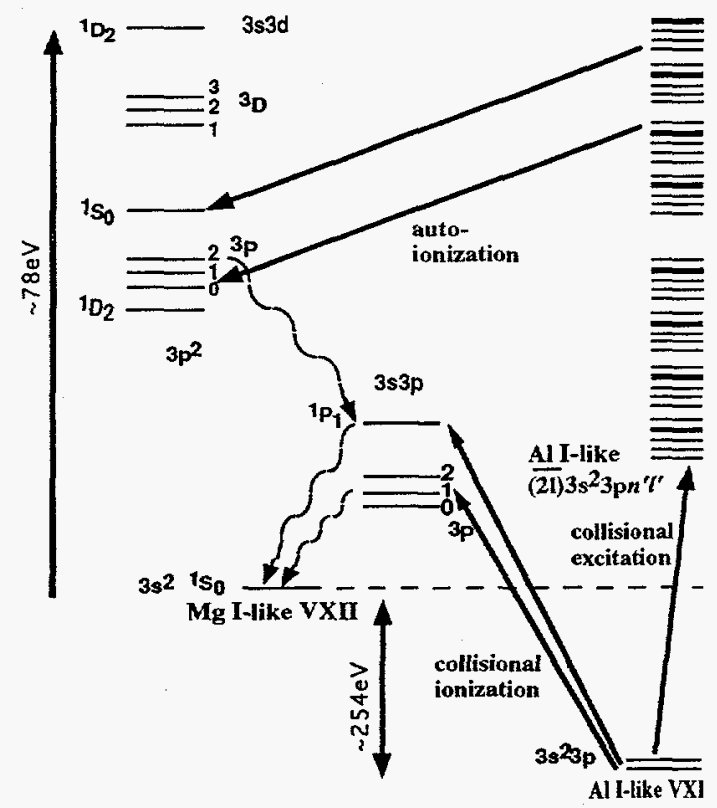

Figure 1. VXII energy levels and key transitions

\section{Observations and Results}

The results of the present models are seen in Table 1. The temperature and density conditions for each model have been chosen to match the experimental conditions in the corresponding reference. 
Table 1

Observed and calculated ratio for the emissivity of the $3 s^{2}{ }^{1} S_{0}-3 s 3 p{ }^{1} P_{1}$ over the $3 s^{2}{ }^{1} S_{0}-3 s 3 p{ }^{3} P_{1}$ line. Columns are (from left to right) the observed intensity ratio (from tokamaks and the active solar corona), the ratio calculated with no ionization channels between adjacent charge states, with ionization equilibrium between adjacent charge states, and with ionization channels between adjacent charge states and twice as much of the All-like ion as the MgI-like ion present.

\begin{tabular}{l|c|r|r|r|r}
\hline Ion & IP $(\mathrm{eV})$ & Observed Ratio & No Ionization & Ionization Equil. & $\mathrm{n}_{\text {AlI }: \mathrm{n}_{M g I}=2: 1}$ \\
\hline TiXI & 265 & $11.5^{\mathrm{a}}$ & 26.7 & 16.9 & 11.1 \\
VXII & 308 & $10.7^{\mathrm{a}}$ & 25.6 & 16.0 & 10.5 \\
CrXIII & 354 & $10.0^{\mathrm{a}}$ & 24.4 & 15.7 & 9.9 \\
FeXV & 456 & $11.9^{\mathrm{b}}$ & 22.4 & 16.6 & 10.9 \\
GeXXI & 836 & $8^{\mathrm{c}, \mathrm{d}}$ & 15.5 & 10.4 & 8.7 \\
SeXXIII & 988 & $8.1^{\mathrm{c}, \mathrm{d}}$ & 13.1 & 9.9 & 8.6 \\
MoXXXI & 1728 & $6.2^{\mathrm{e}}$ & 6.3 & 5.8 & 5.6 \\
\hline
\end{tabular}

${ }^{\mathrm{a}}$ Ref. [8] ${ }^{\mathrm{b}}$ Solar measurement, Ref. [7] ${ }^{\mathrm{c}}$ Ref. [9] ${ }^{\mathrm{d}}$ Ref. $[19]^{\mathrm{e}}$ Measured in Alcator C-Mod, 1995

Details of the experiments for $\mathrm{Ti}, \mathrm{V}$ and $\mathrm{Cr}$ have been published [8]. The value of $R / I$ for FeXV is from a measurement on the active solar corona in Ref. [7]. The data specific to the measurements for $\mathrm{Ge}$ and Se have also been published $[9,6]$. The value of $R / I$ for FeXV is from a measurement on the active solar corona in Ref. [7]. The measurement of R/I for MoXXXI made in the C-Mod tokamak for the present work had $T_{e} \approx 1.6 \mathrm{keV}$ and $\mathrm{N}_{e} \approx 1.0 \times 10^{14} \mathrm{~cm}^{-3}$.

Observations indicate that, in every case, the emitting ion is localized to a region of the plasma with an electron temperature very nearly equal to the ionization potential of the MgI-like ion. The result of introducing the ionization channels into the model is a significant increase in the strength of the intercombination line of the lighter elements in this study. This occurs because the ionization channels (both the resultant cascades from the excitation-autoionization channels and the direct ionization channels) have a much larger relative contribution to the net flux into the $3 \mathrm{~s} 3 \mathrm{p}^{3} \mathrm{P}_{1}$ level than to the flux into the ${ }^{1} \mathrm{P}_{1}$ level.

The agreement between the models with twice as much AlI-like ion as MgI-like ion present and the observations made for $\mathrm{Ti}, \mathrm{V}$ and $\mathrm{Cr}$ is excellent; the conclusion drawn is that the ions are swept inward to a region of the tokamak plasma with a high temperature faster than they are ionized; this is consistent with transport studies done on TFR. MoXXXI, the heaviest ion in this paper, exists in the core of the (C-Mod) tokamak. In this case, MoXXXI ions can not be swept inward, and hence, the ionization equilibrium model is adequate for predicting the $\mathrm{R} / \mathrm{I}$ ratio.
The QSS-C̄ models reveal that the feeding of the intercombination line comes primarily from radiative cascades from the $J=2$ levels of the $3 p^{2}$ and 3s3d configurations (see Fig. 1). In VXII, for example, in the model with ionization equilibrium enforced between adjacent charge states (column three of Table 1), $85 \%$ of the flux into the $3 \mathrm{~s} 3 \mathrm{p}$ ${ }^{3} \mathrm{P}_{1}$ level comes from radiative cascades, $10 \%$ of the flux is from collisional excitation directly from the ground state, and the rest of the flux is from the direct, impact ionization from the AlI-like ion. In SeXXIII, the cascades and excitation channels account for $55 \%$ and $40 \%$, respectively of the flux into the $3 \mathrm{~s} 3 \mathrm{p}{ }^{3} \mathrm{P}_{1}$ state. The high strength of the line is partly due to wavefunction mixing effects; as the ionization state increases, the wavefunctions of the MgI-like 3s3p configuration begin to assume a $j j$-characteristic. Hence, the numbers in Table 1 decrease down the columns; the intercombination line is becoming "less" forbidden.

We identify in Table 2 the $3 \mathrm{~s} 3 \mathrm{p}{ }^{1} \mathrm{P}_{1}-3 \mathrm{p}^{2}{ }^{3} \mathrm{P}_{2}$ line for $\mathrm{Ti}, \mathrm{V}$ and $\mathrm{Cr}$. Observations made in the TFR tokamak [18] during injections of $\mathrm{Ti}, \mathrm{V}$ and $\mathrm{Cr}$ find lines with the same temporal behavior as the MgI-like intercombination line, and which are emitted from the same spatial position in the plasma. Based on the temporal and spatial behavior of these lines, and based on $a b$ initio predictions for the wavelengths of these transitions, we now identify them as new intercombination lines. The right-most column in Table 2 shows the predicted ratio of the brightness of the $3 s^{2}{ }^{1} \mathrm{~S}_{0}-3 \mathrm{~s} 3 \mathrm{p}$ ${ }^{1} \mathrm{P}_{1}$ resonant line over the brightness of the new intercombination lines.

Due to the slow nature of triplet-singlet tran- 
Table 2

Measured and predicted wavelengths for the $3 s 3 p{ }^{1} P_{1}-3 p^{2}{ }^{3} P_{2}$ transitions in MgI-like ions. Columns contain, respectively, the observed wavelength and the wavelength as calculated by RELAC. The last column has the predicted R/I* ratio from the QSS-CR models.

\begin{tabular}{l|r|r|r}
\hline Ion & $\lambda_{\text {obs. }}(\AA)$ & $\lambda_{\text {theor. }}(\AA)$ & $\mathrm{R} / \mathrm{I}$ \\
\hline TiXI & $625.9 \pm 0.2^{\mathrm{a}}$ & 625.24 & 1000 \\
VXII & $570.3 \pm 0.6^{\mathrm{a}}$ & 568.32 & 900 \\
CrXIII & $522.2 \pm 1.2^{\mathrm{a}}$ & 519.37 & 800 \\
FeXV & & 438.55 & 800 \\
GeXXI & & 282.98 & 400 \\
SeXXIII & & 247.80 & 450 \\
MoXXXI & & 152.57 & 800 \\
\hline a Ref. [18]
\end{tabular}

sitions, the $3 \mathrm{~s} 3 \mathrm{p}{ }^{1} \mathrm{P}_{1}-3 \mathrm{p}^{2}{ }^{3} \mathrm{P}_{2}$ line (I*) is not expected to be observable in light elements in any plasma source. However, the $3 \mathrm{p}^{2}{ }^{3} \mathrm{P}_{2}$ state has a significant admixture of the $3 \mathrm{~s} 3 \mathrm{~d}{ }^{1} \mathrm{D}_{2}$ and the $3 \mathrm{p}^{2}{ }^{1} \mathrm{D}_{2}$ levels. For VXII, for example, $3 \mathrm{p}^{2}{ }^{3} \mathrm{P}_{2}$ has $11 \%$ of its amplitude from $3 \mathrm{p}^{2}{ }^{1} \mathrm{D}_{2}$ and $1.5 \%$ from $3 \mathrm{~s} 3 \mathrm{~d}^{1} \mathrm{D}_{2}$. The result is that the upper state, $3 \mathrm{p}^{2}{ }^{3} \mathrm{P}_{2}$, has a strong singlet characteristic, and is fed by an enhanced collisionality with the ground state (which arises from the 3 s3d states which mix with the $3 \mathrm{p}^{2}$ levels). The singlet characteristic also allows the $3 \mathrm{p}^{2}{ }^{3} \mathrm{P}_{2}$ level to have an 'enhanced' decay rate to the $3 \mathrm{~s} 3 \mathrm{p}{ }^{1} \mathrm{P}_{1}$ level. The expected $R / I *$ ratio for the $3 s 3 p{ }^{1} P_{1}-3 p^{2}{ }^{3} P_{2}$ lines in Table 2 are very approximate. A treatment of the cascades from the MgI-like levels of the $3 / 4 l$ ' configurations for $l=p$ and $d$, as well as further study of the wavefunctions of the $3 \mathrm{p}^{2}$ states is needed; these issues will be addressed in a forthcoming work.

\section{Conclusion}

The present work offers models of the $\mathrm{R} / \mathrm{I}$ ratio in several MgI-like ions. The models find the contribution to the emissivity of the intercombination line from autoionizing states of the AlI-like ion is insignificant. In the future, $a b$ initio calculations of direct, impact ionization rates may further improve the agreement between observations and models for the R/I ratio. Also, new intercombination lines between excited states, which are enabled by wavefunction mixing, are identified in the present paper. Work supported under the auspices of U. S. Department of Energy under contract W-7405-ENG-48.

\section{REFERENCES}

1. R. Cowan and K. Widing, ApJ., 180, (1973) 285.

2. W. Behring, L. Cohen, U. Feldman and G. Doschek, ApJ., 203 (1976) 521.

3. A. K. Bhatia and S. O. Kastner, Solar Phys., 65 (1980) 181.

4. R. Christensen, D. Norcross and A. Pradhan, Phys. Rev. A, 32 (1985) 93.

5. P. L. Dufton, A. E. Kingston and K. G. Widing, ApJ, 353 (1990) 323.

6. M. Finkenthal, B. Stratton, H. W. Moos, A. Bar-Shalom, M. Klapisch, Phys. Lett., 108A (1985) 71.

7. U. Feldman, J. Laming, P. Mandelbaum, W. Goldstein and A. Osterheld, ApJ, 398 (1992) 692.

8. M. Finkenthal, R. Bell, H. W. Moos and TFR Group, Phys. Lett., 88A (1982) 165.

9. M. Finkenthal, E. Hinnov, S. Cohen and S. Suckewer, Phys. Lett., 91 (1982) 284.

10. M. Arnaud and J. Raymond, ApJ, 398 (1992) 394.

11. K. Fournier, M. Cohen, W. Goldstein, A. Osterheld, J. Terry, J. Rice, M. Finkenthal, M. May, Manuscript in preparation (1995).

12. V. A. Boiko, I. Skobelev, A. Faenov, Sov. J. Plasma Phys. 10, (1984) 82.

13. K. Fournier, W. Goldstein, D. Pacella, R. Bartiromo, M. Finkenthal and M. May, UCRLJC-121176 June, 1995 (submitted to Phys. Rev. E).

14. M. Klapisch, Comput. Phys. Commun., 2 (1971) 239 . M. Klapisch, J. L. Schwob, B. S. Fraenkel and J. Oreg, J. Opt. Soc. Am., 67 (1977) 148.

15. A. Bar-Shalom, M. Klapisch and J. Oreg, Phys. Rev. A, 38 (1988) 1773.

16. J. Oreg, W. Goldstein and M. Klapisch, Phys. Rev. A, 44 (1991) 1750 .

17. W. Lotz, Z. Phys., 216 (1968) 241. W. Lotz, Z. Phys., 232 (1970) 101.

18. R. Bell, Ph.D. Thesis at JHU, 1982.

19. B. Stratton, Ph.D. Thesis at JHU, 1984. 